\title{
Deletion of Murine Gammaherpesvirus Gene M2 in AID-Expressing B Cells Impairs Host Colonization and Viral Reactivation
}

Shana M. Owens ${ }^{1}$, Darby G. Oldenburg ${ }^{2}$, Douglas W. White ${ }^{2}$, and J. Craig Forrest ${ }^{1,3,4}$

${ }^{1}$ Dept. of Microbiology and Immunology and Center for Microbial Pathogenesis and Host

Inflammatory Responses, University of Arkansas for Medical Sciences, Little Rock, AR

${ }^{2}$ Gundersen Health System, La Crosse, Wisconsin

${ }^{3}$ Winthrop P. Rockefeller Cancer Institute, Little Rock, AR

${ }^{4}$ lead contact:

J. Craig Forrest, Dept. of Microbiology and Immunology, University of Arkansas for Medical Sciences, 4301 W. Markham, Slot 511, Little Rock, AR 72205

Phone: (501) 526-7141, Fax: (501) 686-5359, Email: JCForrest@uams.edu

\section{Running title: Requirement of MHV68 M2 in AID-expressing cells}




\section{ABSTRACT}

Gammaherpesviruses (GHVs) are DNA tumor viruses that establish life-long, chronic

3 infections in lymphocytes of humans and other mammals. GHV infections are associated with

4 numerous cancers, especially in immune compromised hosts. While it is known that GHVs utilize

5 host germinal center (GC) B cell responses during latency establishment, an understanding of

6 how viral gene products function in specific B cell subsets to regulate this process is incomplete.

7 Using murine gammaherpesvirus 68 (MHV68) as a small-animal model to define mechanisms of

8 GHV pathogenesis in vivo, we generated a virus in which the $M 2$ gene was flanked by loxP sites

9 (M2.loxP), enabling the use of Cre-lox technology to define M2 function in specific cell types in

10 infection and disease. The $M 2$ gene encodes a protein that is highly expressed in GC B cells that

11 promotes plasma cell differentiation and viral reactivation. M2 was efficiently deleted in Creexpressing cells, and the presence of loxP sites flanking $M 2$ did not alter viral replication or latency

13 in mice that do not express Cre. In contrast, M2.loxP MHV68 exhibited a deficit in latency

14 establishment and reactivation that resembled M2-null virus, following intranasal (IN) infection of

15 mice that express Cre in all B cells (CD19-Cre). Nearly identical phenotypes were observed for

16 M2.loxP MHV68 in mice that express Cre in germinal center (GC) B cells (AID-Cre). However,

17 neither colonization of draining lymph nodes after IN infection nor the spleen after intraperitoneal

18 (IP) infection required M2, although the reactivation defect was retained. Together, these data

19 confirm that M2 function is B cell-specific and demonstrate that M2 primarily functions in AID-

20 expressing cells to facilitate MHV68 dissemination to distal latency reservoirs within the host and

21 reactivation from latency. Our study reveals that a viral latency gene functions within a distinct

22 subset of cells to facilitate host colonization. 


\section{IMPORTANCE}

24 Gammaherpesviruses establish life-long chronic infections in cells of the immune system

25 that can lead to lymphomas and other diseases. To facilitate colonization of a host,

26 gammaherpesviruses encode gene products that manipulate processes involved in cellular

27 proliferation and differentiation. Whether and how these viral gene products function in specific

28 cells of the immune system is poorly defined. We report here the use of a viral genetic system

29 that allows for deletion of specific viral genes in discrete populations of cells. We employ this

30 system in an in vivo model to demonstrate cell-type-specific requirements for a particular viral

31 gene. Our findings reveal that a viral gene product can function in distinct cellular subsets to direct

32 gammaherpesvirus pathogenesis. 


\section{INTRODUCTION}

Gammaherpesviruses (GHVs) are DNA tumor viruses that include the human pathogens Epstein-Barr virus (EBV) and Kaposi sarcoma-associated herpesvirus (KSHV). GHVs are lymphotropic viruses with a biphasic infection cycle that is characterized by two distinct stages: productive Iytic replication or chronic, life-long latency (1). Lytic replication is characterized by temporally regulated viral gene expression, replication of viral DNA, and production of infectious viral progeny (1). After resolution of acute infection, latency is established and viral genomes are maintained within germinal center (GC) and memory B cells (2). During latency, viral gene expression is restricted to a few latency gene products and non-coding RNAs $(3,4)$. These molecules function to manipulate host-cell physiology, maintain viral genomes, and thwart

43 immune surveillance (5-10). In response to specific stimuli, latent viral genomes can reinitiate the

44 lytic cycle, a process called reactivation (11). Reactivation enables infection of new hosts and is thought to help maintain latent reservoirs within a chronically infected host $(11,12)$.

To define the processes used by GHVs to infect a living organism and cause disease, we

47 and others utilize the natural rodent pathogen murine gammaherpesvirus 68 (MHV68, also known

48 as murid herpesvirus 4 or MuHV-4) (12). The MHV68 genome is co-linear with EBV and KSHV,

49 and like other GHVs, MHV68 preferentially infects B lymphocytes and can cause cancer, 50 especially in immune compromised animals $(13,14)$. Because MHV68 readily infects and 51 establishes chronic infection in inbred, outbred, and genetically modified mouse strains $(11,15)$, 52 MHV68 serves as a tractable small animal model for studying the function of viral gene products 53 and the host immune response to GHV infections.

During latency establishment GHVs are thought to augment and usurp the GC reaction

$55(11,16,17)$. The GC reaction is a B cell maturation process that is characterized by rapid cellular 56 proliferation and the accumulation of mutations in the immunoglobulin locus, which encodes the 57 B cell receptor and antibodies, and is mediated by the enzyme activation-induced cytidine 58 deaminase (AID) (18-20). Subversion of the GC response by GHVs is thought to expand the 
reservoir of latently infected cells and promote the infection of long-lived memory B cells (MBCs) (21). While it is well known that GHVs establish and maintain chronic infection within GC B cells, requirements for specific viral gene products in this process are still being investigated.

The MHV68 M2 gene is highly expressed in GC B cells and, while M2 has no known viral or cellular homologs at the sequence level, it is hypothesized to be a functional homolog of the EBV latent membrane protein 2A (LMP2A) and the KSHV K1 protein (22). Transcription of M2 within B cells is driven by multiple closely-linked promoters, which generate two spliced and one unspliced M2 transcript. The spliced transcripts encode the M2 protein and are apparently regulated in a B cell-specific manner, as spliced transcripts are not detected during lytic infection, whether in cell culture or the lung epithelium $(4,23)$. The M2 protein functions as a scaffold that interacts with membrane-associated signaling molecules to mimic B cell-receptor activation and promote calcium-mediated activation of the nuclear factor of activated T cells (NFAT) pathway (24). NFAT activation driven by M2 induces the plasma cell-associated transcription factor, interferon regulatory factor 4 (IRF4), which enforces a gene expression program involved in plasma cell differentiation and promotes production of anti-inflammatory cytokines, such as IL-10

74 (10, 24). Studies using M2-null MHV68 (M2.Stop) and viruses with specific mutations in putative signaling residues demonstrated that M2 facilitates latency establishment in the spleen after IN, but not IP, inoculation of mice (25-27). M2 is also generally required for viral reactivation from

77 latency in vivo, a phenotype that correlates with reduced plasma cell infection by M2-null virus

78 (28). Forced retroviral expression of M2 in primary murine B cells in tissue culture and following

79 in vivo transfer drives B cells toward a GC B cell phenotype, which ultimately differentiate, into 80 plasma cells $(28,29)$. While detection of spliced $M 2$ transcripts in B cells, but not other cell types, 81 suggests a B cell-specific function, distinct requirements for M2 in unique B cell subsets during 82 viral colonization of the host are not defined.

83 Since GC B cells are critical early targets for GHV infection, determining how specific viral 84 gene products function within these cells to permit latency is fundamental to understanding GHV 
pathogenesis. We previously reported development of a viral genetic platform that allows for dissection of cell-type-specific roles of viral gene products in vivo (30). Building from this technology, we engineered a recombinant MHV68 in which the gene encoding M2 was flanked by loxP sequences (floxed; $M 2 . l o x P$ ) to enable the conditional deletion of $M 2$ in cells that express Cre recombinase. We used this system to define the function of M2 in specific cell-types in mice, especially GC B cells, during latency establishment by MHV68.

\section{RESULTS}

\section{Generation and validation of M2.IoxP MHV68}

Teasing apart the function of GHV gene products in vivo is complicated in part by the biphasic infection cycle, as well as the convoluted dissemination process required to establish splenic latency. Traditional methods of viral mutagenesis result in complete ablation of a gene of interest. This approach discounts the role of multi-functional proteins that are required in different phases of infection or within distinct cell-types. M2 encodes a latency-specific gene product that facilitates MHV68 establishment of latency and reactivation and promotes B cell differentiation in infected mice $(3,27-29)$. To better define roles for the MHV68 latency gene product M2 within distinct populations of B lymphocytes, we generated a recombinant virus to enable cell-typespecific deletion of the M2 gene in infected cells that express Cre recombinase. We previously demonstrated the feasibility and utility of this approach in a study that defined B cell-specific requirements for ORF73, the gene that encodes the MHV68 latency-associated nuclear antigen (mLANA) (30). In the new MHV68 recombinant, loxP sites were inserted flanking the $M 2$ gene within the MHV68 BAC, and viral stocks were generated (M2.loxP MHV68, Fig 1A). Appropriate BAC modification was confirmed by restriction fragment length polymorphism analysis (RFLP, Fig 1B) 109 and sequencing of the targeted genetic locus. To confirm that floxed $M 2$ was efficiently deleted in 110 the presence of Cre, we infected either Vero or Vero-Cre cells with WT MHV68, M2.Stop, or 
111 M2.loxP, and then evaluated M2 locus integrity by PCR. Although intact M2 was readily detected

112 in Vero cells infected with M2.loxP, only the M2 deletion amplicon was detected in Vero-Cre cells

113 infected with M2.loxP (Fig 1C). The full-length M2 locus was detected in both cell-types infected

114 by WT MHV68 and M2.Stop. Furthermore, an adjacent viral gene, $M 3$, remained intact when $M 2$

115 was deleted, suggesting that viral gene deletion is correctly targeted and specific (Fig 1C). These

116 results demonstrate that Cre-mediated recombination efficiently deletes the $M 2$ gene from the

117 M2.loxP MHV68 genome in cell culture.

118 To ensure that the presence of IoxP sites flanking M2 did not inadvertently attenuate the 119 virus, we evaluated M2.loxP infection relative to control viruses. In cultured cells, M2.loxP MHV68 120 replicated with similar kinetics to WT MHV68 in both single- and multi-step growth analyses (Fig 121 2A). Further, M2.loxP MHV68 titers were equivalent to WT and M2-null MHV68 on day 7 after IN 122 inoculation in lungs of $\mathrm{C} 57 \mathrm{BL} / 6$ mice, a time point that approximates the peak of acute viral 123 replication in vivo (Fig 2B). This finding confirms that the presence of loxP sites did not impact acute viral replication in vivo, and M2.Stop results further demonstrate that M2 is not required for the insertion of loxP sites flanking the $M 2$ gene does not impair MHV68 latency establishment in or reactivation from the spleen of WT mice.

\section{M2 is required in $C D 19^{+} B$ cells for latency establishment and reactivation}

RT-PCR analyses suggest that a properly spliced $M 2$ transcript capable of yielding the

134 functional M2 protein is only present in B cells $(4,23,31)$. From this observation it is hypothesized 135 that M2 primarily functions in B cells to promote MHV68 latency and reactivation. To more 136 conclusively test this hypothesis, we infected mice that express Cre recombinase under control 
of the B cell-specific CD19 promoter (CD19-Cre) to facilitate M2 deletion in all B cells. On day 7 after IN inoculation, titers of M2.loxP and WT MHV68 were equivalent in lungs (Fig 3A). However, splenomegaly, a consequence of the infectious mononucleosis-like syndrome caused by MHV68, did not occur following infection with M2.loxP (Fig 3B). A PCR analysis of the M2 locus demonstrated that $M 2$ was deleted in these mice (Fig 3C). Latency establishment by M2.loxP

142 also was 10-fold lower than WT MHV68 in spleens of CD19-Cre mice on day 16 post-infection

143 (Fig 3D and Table 1), and M2.loxP reactivation from explanted splenocytes was ca. 100-fold 144 lower than WT MHV68 (Fig 3E and Table 2), indicating a further 10-fold reduction over the latency 145 defect. The deficits exhibited by M2.loxP following infection of CD19-Cre mice appear to 146 phenocopy M2.Stop infection of WT C57BL/6 mice (Fig 2), indicating that M2 primarily functions in B lymphocytes to facilitate latency establishment and reactivation following IN infection.

\section{M2 is required in AID-expressing B cells for MHV68 latency and reactivation}

the pool of long-lived MBCs. At early latency time points the majority of MHV68-infected cells

to the direct role of $\mathrm{M} 2$ in $\mathrm{B}$ cell maturation processes and the importance of $\mathrm{GC} B$ cells during promote latency establishment.

To determine how M2 functions within the GC B cell compartment, we evaluated M2.loxP infection in mice that express Cre recombinase under control of the promoter for the activationinduced cytidine deaminase (AID), the enzyme responsible for somatic hyper-mutation (SHM) 160 and class-switch recombination (CSR) in GC B cells (19). Following IN infection of AID-Cre mice, 161 M2.loxP viral titers in the lung were equivalent to WT MHV68 on day 7 post-infection (Fig 4A). A 162 PCR analysis of the M2 locus demonstrated that M2 was deleted in these mice (Fig 4C). Similar 
163

164

to results in CD19-Cre mice, M2.IoxP caused reduced splenomegaly relative to WT virus on day 16 post-infection (Fig 4B and Table 1), and M2.loxP latency in the spleen was 10-fold lower than WT MHV68 at this time point (Fig 4D). Additionally M2.loxP reactivation was below the limit of detection of 1 in 100,000 cells (Fig 4E and Table 2), which indicates that M2 expression in AIDexpressing B cells potentiates MHV68 reactivation. These data strongly suggest that M2 expression within the GC compartment drives efficient latency establishment in and reactivation from the spleen following intranasal infection.

\section{M2 is not required within GC B cells for colonization of lymphoid tissue}

Following IN inoculation, MHV68 drains from the lungs to the mediastinal lymph nodes (MLNs) (32). From MLNs the virus disseminates via the blood to distal latency reservoirs, such as the spleen (33). We previously demonstrated that ORF73, the viral gene that encodes the MHV68 LANA homolog, is not necessary in $\mathrm{CD}_{1}{ }^{+} \mathrm{B}$ cells for initial viral deposition in MLNs, despite its critical importance in hematogenous dissemination (30). Whether M2 is required for MLN infection and its requirement in specific B cell subsets has not been tested. We therefore evaluated MLN infection for M2.loxP, M2.Stop and WT MHV68 in either C57BL/6 or AID-Cre mice. In C57BL/6 mice, all three viruses were detected at equivalent levels in MLNs on day 16 after IN infection. In agreement with these findings, M2.loxP also established latency at levels similar to WT MHV68 in the MLNs of AID-Cre mice (Fig 5A and 5B). These data support the conclusion that M2 is not necessary for latency in draining lymphoid tissue. Given that latency in the spleen is reduced in the absence of M2 following IN inoculation, we hypothesize that MHV68 facilitates viral dissemination to the spleen after initial seeding of the draining MLN.

Intraperitoneal (IP) inoculation with MHV68 is thought to provide direct viral access to the spleen, bypassing any requirements for trafficking that may impact latency establishment following IN inoculation (27, 34). Similar to observations for MLNs, M2-null MHV68 achieves normal levels of latency in the spleen after IP inoculation, but still exhibits a reactivation defect 
$(27,28,35)$. To determine how deletion of $M 2$ from AID-expressing B cells influenced chronic MHV68 infection after IP inoculation, we compared infections of M2.loxP and WT MHV68 in AIDCre mice. Analogous to observations following IN inoculations, M2.loxP exhibited reduced 192 splenomegaly compared to WT virus following IP infection of AID-Cre mice (Fig 6A). While the 193 frequency of cells harboring latent M2.loxP genomes in spleens of AID-Cre mice was only slightly 194 reduced compared to WT MHV68, M2.loxP was severely impaired in its capacity to reactivate

195 from splenic latency (Fig 6B and 6C). In peritoneal exudate cells (PECs), M2.loxP and WT MHV68 196 latency and reactivation were equivalent, which is consistent with the notion that MHV68 does not 197 use AID-expressing cells to access or reactivate from cells present in the peritoneum (Fig 6D and 198 6E). We also infected CD19-Cre mice as a control to confirm that phenotypes were similar when M2 was deleted in B cells in general. Again, M2.loxP established latency in the spleen at levels similar to WT MHV68, but still exhibited a reduction in reactivation (Fig 6F and 6G). Reduced detection of the $M 2$ locus in M2.loxP-infected AID-Cre and CD19-Cre mice indicates that M2 was efficiently deleted from both strains after IP infection (Fig 6H). Together, these data suggest that M2 is not required for viral latency establishment within primary lymphatic tissues, but functions after deposition in draining lymph nodes to promote viral dissemination to the spleen. (28). M2 expression in BCL-1 lymphoma cell lines promotes features of plasmacyte morphology, including increased size, granularity, and secretion of high levels of IgM. The notion that M2 drives 210 plasmablast differentiation is consistent with reports that M2.Stop virus persists in B lymphocytes 211 which have not undergone class-switching $\left(\lg D^{+} \operatorname{lgG} 2 a^{-}\right)(28,36)$. To determine whether $M 2$ 212 deletion from AID-expressing cells influences infection of plasmablasts in the spleen, we sorted $213 \mathrm{CD} 138^{+} / \mathrm{CD}^{\mathrm{lo}}$ cells from spleens of infected AID-Cre mice (37) (Fig 7A) and performed qPCR 214 to quantify viral genomes. Interestingly, by this method of quantification, we observed a 4.5 cycle 
215 difference, indicative of a ca. 20-fold deficit, in M2.loxP genome levels compared to WT virus in

216 unsorted cells (Fig 7B). However, M2.loxP DNA in the plasmablast population was essentially

217 equivalent to WT MHV68 (Fig 7C). We suspect that the larger impact on the general level of viral

218 genomes detected in this experiment reflects that qPCR provides an absolute quantification of

219 viral genomes in the population, rather than the evaluation of cells with at least one genome that

220 is provided by the standard LD-PCR approach. This suggests that, while similar numbers of cells

221 harbor MHV68 genomes (Fig 6), there are fewer viral genomes per cell upon deletion of M2 from

222 AID-expressing cells. In contrast, the absence of a severe phenotype for plasmablast infection

223 suggests that infection of plasmablasts is either less dependent on M2 or does not require prior

224 infection of an AID-expressing cell.

\section{DISCUSSION}

Gammaherpesviruses have evolved to potently manipulate and usurp immune responses generated toward viral antigens. For instance, studies in SAP- and IL-21 receptor-deficient mice demonstrate that MHV68 utilizes the general GC response to its own infection as a means to expand the latent cellular reservoir and generate long-lived reservoirs like $\operatorname{MBCs}(21,38,39)$.

231 Understanding how specific viral genes engage these immune pathways is important for 232 understanding the basic biology of GHV infection. Through the generation of null and point mutant 233 viruses, overexpression studies, and utilization of fluorescent tagging, the MHV68 M2 protein is 234 already linked to manipulation of B cell activation and differentiation (22, 27-29, 35); but when and 235 where M2 exerts its functions to facilitate chronic viral infection and reactivation from latency are 236 not known. To better define M2 functions in MHV68 pathogenesis, we describe here the 237 generation and in vivo characterization of M2.loxP, a recombinant virus with the capacity to 238 conditionally delete the M2 locus in a Cre-dependent manner. Using this system, we demonstrate 239 that loss of M2 from AID-expressing B cells essentially recapitulates M2-null virus phenotypes in 
latency establishment and reactivation. We therefore conclude that M2 primarily functions within the GC B cell compartment to facilitate chronic MHV68 infection (Fig 8).

While our results indicate that the presence of loxP sites flanking $M 2$ in the MHV68 genome does not overtly influence lytic replication and latency, there are potential caveats to 244 consider. The effect of loxP insertion on adjacent gene expression has not been assessed. The 245 coding capacity of the MHV68 genome is densely packed $(40,41)$, and it is possible that loxP 246 insertion or deletion of the locus inadvertently disrupts the function of overlapping or adjacent 247 transcripts. To better characterize the potential off target effects that could occur following M2 248 deletion, further analysis such as single-cell RNA sequencing is required. Additionally, if paired 249 with mechanisms for marking infected cells, such as expression of a fluorescent or enzymatic 250 reporter $(2,42)$, the impact of $M 2$ deletion on downstream infection of specific cell populations could be evaluated. Moreover, a marking approach could further enhance evaluations of M2 functions in signal transduction (24, 43-45) and impacts on cellular transcription (24), especially manipulation of the germinal center response. As mentioned earlier, M2-null MHV68 accumulates

254 in naïve B cells during latency establishment and does not infect plasma cells $(28,36)$. Our qPCR data following sorting of plasmablast populations suggests that either MHV68 passage through AID-expressing cells only minimally influences plasmablast infection or that these cells are directly

257 infected by the virus. Use of marking approach as described above to specifically isolate the 258 infected cells could enhance attempts to further phenotype the infected plasmablast population, 259 and infection of mice that express Cre in plasma cells could also be performed in future 260 experiments.

MHV68 colonization of the host is thought to require viral dissemination from primary sites

262 of infection into draining lymph nodes, initial expansion in the lymph node, and subsequent relay

263 via the blood to secondary lymphoid organs, such as the spleen $(11,30,32)$. Our data with both

264 M2.Stop and M2.loxP demonstrate that M2 is not required for MHV68 to reach the draining lymph 265 nodes. Similarly, using IP infection to bypass trafficking requirements for colonization of the 
spleen, both M2-null $(27,35,36)$ and M2.loxP MHV68 efficiently establish latency in the spleen,

though viral reactivation is severely impaired. These findings suggest that M2 is not directly required for MHV68 to establish latency in lymphoid tissue, but is required for downstream colonization events. Since M2.IoxP accumulates to levels that are equivalent to WT virus in the MLN after IN infection of AID-Cre mice, but does not efficiently infect the spleen, it is reasonable to conclude that MHV68 traffics through a GC B cell to facilitate the seeding of distal latency reservoirs. Clearly this is not absolute, as viral genomes are still detected in the spleen following

273 IN infection of Cre-expressing mice. M2.loxP defect corresponds to the accumulation of M2.Stop virus in naïve B cells, since M2 would presumably still be present in naïve B cells, due to a lack of AID-expression (46). M2-driven reactivation facilitating seeding of the spleen is possible, however another virus in which we conditionally delete ORF50, the gene that encodes the replication and transcription activator (RTA), suggests that reactivation/lytic replication in B cells is not required for splenic latency after IN inoculation (manuscript in preparation). We speculate that M2's function in augmenting signaling within GC B cells is critical to enhancing MHV68 trafficking to secondary sites of latency in the mouse. We anticipate pairing cell-type-specific ablation of M2 function with single-cell transcriptomic and proteomic approaches in future experiments to evaluate these possibilities.

\section{MATERIALS AND METHODS}

Ethics statement. Mouse experiments were carried out in accordance with National Institutes of Health, United States Dept. of Agriculture, and UAMS Division of Laboratory Animal Medicine and Institutional Animal Care and Use Committee (IACUC) guidelines. The protocol supporting this study was approved by the University of Arkansas for Medical Science (UAMS) IACUC (animal use protocol 3817). Mice were anesthetized prior to inoculations and sacrificed humanely at the 291 end of experiments. 
Cells and viruses. NIH 3T12 (ATCC CCL-164), 3T12 FIp+ (30), BHK21 (ATCC CCL-10), Vero (ATCC CCL-81), Vero-Cre (47), were cultured in Dulbecco's Modified Eagle Medium (DMEM) supplemented with $10 \%$ fetal bovine serum (FBS), $100 \mathrm{U} / \mathrm{ml}$ penicillin, $100 \mathrm{ug} / \mathrm{ml}$ streptomycin, and $2 \mathrm{mM} \mathrm{L-glutamine} \mathrm{(cDMEM).} \mathrm{Cells} \mathrm{were} \mathrm{maintained} \mathrm{at} 37^{\circ} \mathrm{C}$ in $5 \% \mathrm{CO}_{2}$ and $\sim 99 \%$ humidity. Murine embryonic fibroblasts (MEFs) were harvested from C57BL/6 mouse embryos as previously described (34). Previously described viruses used in this study include FRT BAC MHV68 (WT MHV68) (30) and M2.null MHV68 (M2.STOP) (27). of $\mathrm{M} 2$ in a FRT BAC template by two successive rounds of en passant mutagenesis as

301 previously described (30) utilizing primers:

M2loxpUP_for

5' - GTCTGTCACGCTTCTCCTTCCAGGCGTGTTTAAAGAAAAAATAACTTCGTATAGCATAC ATTATACGAAGTTATGTTATGTTCTGCGTTAGCACTAGGGATAACAGGGTAATCGATTT - 3’ M2loxpUP_rev 5' - GGTTAATTGGTTGTAACACTGGCCAGGCGTGTTTAAAGAAAAAATAACTTCGTATAGC ATACATTATACGAAGTTATGTTATGTTCTGCGTTAGCACCTTCACTGTTACTCCTCGCC - 3' M2loxpDWN_for 5' - GTGGGgGTGTTGgGgCCATTACCTGAAAACGAAACCTCATATAACTTCGTATAGCATA CATTATACGAAGTTATCATCAGACTAGTGCCTGTTGTAGGGATAACAGGGTAATCGATTT-3'

311 M2loxpDWN_rev GCTATACGAAGTTATATGAGGTTTCGTTTTCAGGTGCCAGTGTTACAACCAATTAACC - 3' .

314 Viruses were passaged in Flp-expressing 3T12 fibroblasts to remove the BAC cassette, and 315 titers were quantified as previously described (30).

316 Mouse infections and tissue harvests. Male and female, C57BL/6, CD19-Cre (B6.129P2(C)$317 \mathrm{Cd19tm1}(\mathrm{cre}) \mathrm{Cgn} / \mathrm{J})$, and AID-Cre (B6.129P2-Aicdatm1(cre)Mnz/J) were purchased from 
Jackson Laboratories. Mice were bred and maintained according to all local, state, and federal guidelines under the supervision of the UAMS Division of Laboratory Animal Medicine. Eight-toten-week-old mice were anesthetized using isoflurane and inoculated with 1000 PFU of viruses diluted in incomplete DMEM $(20 \mu \mathrm{l})$ for IN inoculations or injected with 1000 PFU of virus diluted in incomplete DMEM (200 ul) for IP inoculations. Splenocytes and peritoneal exudate cells (PECs) were harvested as previously described (48). Cells from draining lymph nodes were isolated as previously described (30).

Splenocyte isolation and limiting-dilution analyses. Spleens were homogenized in a tenBroek tissue disrupter. Red blood cells were lysed by incubating tissue homogenate in $8.3 \mathrm{~g} / \mathrm{L}$ ammonium chloride for 10 minutes at room temperature with shaking. Cells were filtered through $40 \mu \mathrm{M}$ mesh to reduce clumping. Frequencies of cells harboring MHV68 genomes were determined using LD-PCR analysis as previously described (49). Frequencies of latently-infected cells capable of reactivating were determined using a limiting-dilution analysis for cytopathic effect induced on an indicator BHK21 monolayer as previously described (34).

Plaque Assays. Plaque assays were performed as previously described using BHK21 cells ( $2 \mathrm{x}$ $10^{5}$ cells/well). Briefly, infected cells were overlaid with $1.5 \%$ methylcellulose in DMEM supplemented with $2.5 \%$ calf serum, $100 \mathrm{U} / \mathrm{ml}$ penicillin, $100 \mu \mathrm{g} / \mathrm{ml}$ streptomycin, and $2 \mathrm{mM} \mathrm{L-}$ glutamine, and incubated at $37^{\circ} \mathrm{C}$ for $4-6$ days. Cell monolayers were stained with a solution of crystal violet in formalin for identification and quantification of plaques.

337 Cell Sorting. Splenocytes were isolated and sorted by flow cytometry. Blocking and detection 338 antibodies were diluted in PBS with BSA and EDTA. Splenocytes were blocked with anti-CD16/32 339 (BD Biosciences) for 15 mins at $4^{\circ} \mathrm{C}$ prior to surface staining for 30 mins in the dark at $4^{\circ} \mathrm{C}$. Dead 340 cells were labeled using Fixable Viability Dye eFlour 780 (eBioscience) as per manufacturer's 341 instructions. Surface stains include: dump gate (CD3, Ter119, CD11b, CD11c-PerCp5.5), B220342 redFluor 710 (Tonbo), CD19-BV650, CD38-PE-Cy7, CD138-PE (Biolegend). Cells were sorted 343 on a FACS Aria using FACS Diva software. Data was analyzed using FlowJo software (v10.6.2). 
344 Nucleic acid isolation and qPCR. DNA was isolated by phenol/chloroform extraction and

345 ethanol precipitation. $50 \mathrm{ng}$ of DNA was diluted in PowerUp SYBR Green Master Mix

346 (ThermoFisher) and analyzed by quantitative real-time PCR in an Applied Biosystems

347 QuantStudio thermocycler. Cycling conditions were $10 \mathrm{~min}$ at $95^{\circ} \mathrm{C}$ followed by 40 cycles of $15 \mathrm{~s}$

348 at $95^{\circ} \mathrm{C}$ and $1 \mathrm{~min}$ at $60^{\circ} \mathrm{C}$, using primers specific to the viral ORF72 gene or cellular GAPDH

349 gene $(3,50)$. Biological triplicate samples were analyzed in technical duplicate using the $\triangle \triangle C T$

350 comparative threshold cycle method as previously described (50). Fold change in ORF72 levels

351 for M2.loxP was calculated relative to WT MHV68.

\section{ACKNOWLEDGEMENTS}

354 We thank the laboratory of Dr. Jason Stumhofer for assistance in flow cytometry antibody panel 355 design and Andrea Harris in the UAMS Flow Cytometry Core. This work was supported in part by 356 R01CA167065 of the NIH National Cancer Institute and start-up funds from the UAMS College of 357 Medicine and Arkansas Biosciences Institute to J.C.F. The Flow Cytometry Core and work 358 described here also was supported in part by the Center for Microbial Pathogenesis and Host 359 Inflammatory Responses award P20GM103625 from the NIH National Institute of General 360 Medical Sciences Centers of Biomedical Research Excellence. D.W.W. and D.G.O. were 361 supported by the Gundersen Medical Foundation. The funders had no role in study 362 design, data collection and interpretation, or the decision to submit the work for publication. 


\section{REFERENCES}

365 1. Boehmer PE, Nimonkar AV. 2003. Herpes virus replication. IUBMB Life 55:13-22.

366 2. Collins CM, Speck SH. 2012. Tracking murine gammaherpesvirus 68 infection of germinal center B cells in vivo. PLoS One 7:e33230.

3. Virgin HWt, Presti RM, Li XY, Liu C, Speck SH. 1999. Three distinct regions of the murine gammaherpesvirus 68 genome are transcriptionally active in latently infected mice. J Virol 73:2321-32.

4. Martinez-Guzman D, Rickabaugh T, Wu TT, Brown H, Cole S, Song MJ, Tong L, Sun R. 2003. Transcription program of murine gammaherpesvirus 68. J Virol 77:10488-503.

5. Fowler P, Marques S, Simas JP, Efstathiou S. 2003. ORF73 of murine herpesvirus-68 is critical for the establishment and maintenance of latency. J Gen Virol 84:3405-16.

6. Habison AC, Beauchemin C, Simas JP, Usherwood EJ, Kaye KM. 2012. Murine gammaherpesvirus 68 LANA acts on terminal repeat DNA to mediate episome persistence. J Virol 86:11863-76.

7. Bowden RJ, Simas JP, Davis AJ, Efstathiou S. 1997. Murine gammaherpesvirus 68 encodes tRNA-like sequences which are expressed during latency. J Gen Virol 78 ( Pt

8. Gangappa S, van Dyk LF, Jewett TJ, Speck SH, Virgin HWt. 2002. Identification of the in vivo role of a viral bcl-2. J Exp Med 195:931-40.

9. Upton JW, van Dyk LF, Speck SH. 2005. Characterization of murine gammaherpesvirus 68 v-cyclin interactions with cellular cdks. Virology 341:271-83.

385 10. Siegel AM, Herskowitz JH, Speck SH. 2008. The MHV68 M2 protein drives IL-10 dependent B cell proliferation and differentiation. PLoS Pathog 4:e1000039.

387 11. Barton E, Mandal P, Speck SH. 2011. Pathogenesis and host control of gammaherpesviruses: lessons from the mouse. Annu Rev Immunol 29:351-97. 
12. Speck SH, Virgin HW. 1999. Host and viral genetics of chronic infection: a mouse model of gamma-herpesvirus pathogenesis. Curr Opin Microbiol 2:403-9.

13. Olivadoti M, Toth LA, Weinberg J, Opp MR. 2007. Murine gammaherpesvirus 68: a model for the study of Epstein-Barr virus infections and related diseases. Comp Med

14. Fujiwara S, Nakamura H. 2020. Animal Models for Gammaherpesvirus Infections: Recent Development in the Analysis of Virus-Induced Pathogenesis. Pathogens 9.

15. Dong S, Forrest JC, Liang X. 2017. Murine Gammaherpesvirus 68: A Small Animal Model for Gammaherpesvirus-Associated Diseases. Adv Exp Med Biol 1018:225-236.

16. Flano E, Husain SM, Sample JT, Woodland DL, Blackman MA. 2000. Latent murine gamma-herpesvirus infection is established in activated B cells, dendritic cells, and

17. Willer DO, Speck SH. 2005. Establishment and maintenance of long-term murine gammaherpesvirus 68 latency in B cells in the absence of CD40. J Virol 79:2891-9.

18. Mesin L, Ersching J, Victora GD. 2016. Germinal Center B Cell Dynamics. Immunity 45:471-482.

19. Muramatsu M, Kinoshita K, Fagarasan S, Yamada S, Shinkai Y, Honjo T. 2018. Pillars Cytidine Deaminase (AID), a Potential RNA Editing Enzyme. Cell. 2000. 102: 553-563. J

20. Bransteitter R, Pham P, Scharff MD, Goodman MF. 2003. Activation-induced cytidine deaminase deaminates deoxycytidine on single-stranded DNA but requires the action of

412 21. Kim IJ, Flano E, Woodland DL, Lund FE, Randall TD, Blackman MA. 2003. Maintenance development of memory B cells. J Immunol 171:886-92. 
22. Rangaswamy US, O'Flaherty BM, Speck SH. 2014. Tyrosine 129 of the murine gammaherpesvirus M2 protein is critical for M2 function in vivo. PLoS One 9:e105197.

23. DeZalia M, Speck SH. 2008. Identification of closely spaced but distinct transcription initiation sites for the murine gammaherpesvirus 68 latency-associated M2 gene. J Virol

24. Rangaswamy US, Speck SH. 2014. Murine gammaherpesvirus M2 protein induction of IRF4 via the NFAT pathway leads to IL-10 expression in B cells. PLoS Pathog 10:e1003858.

9.

427 26. Simas JP, Marques S, Bridgeman A, Efstathiou S, Adler H. 2004. The M2 gene product of murine gammaherpesvirus 68 is required for efficient colonization of splenic follicles

431 27. Jacoby MA, Virgin HWt, Speck SH. 2002. Disruption of the M2 gene of murine but is not necessary for expansion of latently infected germinal centre B cells. J Gen gammaherpesvirus 68 alters splenic latency following intranasal, but not intraperitoneal,

434 28. Liang X, Collins CM, Mendel JB, Iwakoshi NN, Speck SH. 2009. Gammaherpesvirusdriven plasma cell differentiation regulates virus reactivation from latently infected B

437 29. Terrell S, Speck SH. 2017. Murine gammaherpesvirus M2 antigen modulates splenic B cell activation and terminal differentiation in vivo. PLoS Pathog 13:e1006543. 
30. Salinas E, Gupta A, Sifford JM, Oldenburg DG, White DW, Forrest JC. 2018. Conditional mutagenesis in vivo reveals cell type- and infection stage-specific requirements for

31. Ebrahimi B, Dutia BM, Roberts KL, Garcia-Ramirez JJ, Dickinson P, Stewart JP, Ghazal

32. Frederico B, Milho R, May JS, Gillet L, Stevenson PG. 2012. Myeloid infection links epithelial and B cell tropisms of Murid Herpesvirus-4. PLoS Pathog 8:e1002935.

33. Feldman ER, Kara M, Oko LM, Grau KR, Krueger BJ, Zhang J, Feng P, van Dyk LF, Renne R, Tibbetts SA. 2016. A Gammaherpesvirus Noncoding RNA Is Essential for

34. Weck KE, Barkon ML, Yoo LI, Speck SH, Virgin HI. 1996. Mature B cells are required for acute splenic infection, but not for establishment of latency, by murine

35. Herskowitz JH, Siegel AM, Jacoby MA, Speck SH. 2008. Systematic mutagenesis of the infection. J Virol 82:3295-310.

36. Herskowitz JH, Jacoby MA, Speck SH. 2005. The murine gammaherpesvirus 68 M2 gene is required for efficient reactivation from latently infected B cells. J Virol 79:2261-

37. Tellier J, Nutt SL. 2017. Standing out from the crowd: How to identify plasma cells. Eur J Immunol 47:1276-1279.

38. Collins CM, Speck SH. 2015. Interleukin 21 signaling in B cells is required for efficient establishment of murine gammaherpesvirus latency. PLoS Pathog 11:e1004831.

39. Collins CM, Speck SH. 2014. Expansion of murine gammaherpesvirus latently infected B cells requires T follicular help. PLoS Pathog 10:e1004106. 
40. Virgin HWt, Latreille P, Wamsley P, Hallsworth K, Weck KE, Dal Canto AJ, Speck SH. 1997. Complete sequence and genomic analysis of murine gammaherpesvirus $68 . \mathrm{J}$ Virol 71:5894-904.

41. O'Grady T, Feswick A, Hoffman BA, Wang Y, Medina EM, Kara M, van Dyk LF, Flemington EK, Tibbetts SA. 2019. Genome-wide Transcript Structure Resolution

42. Nealy MS, Coleman CB, Li H, Tibbetts SA. 2010. Use of a virus-encoded enzymatic marker reveals that a stable fraction of memory B cells expresses latency-associated nuclear antigen throughout chronic gammaherpesvirus infection. J Virol 84:7523-34.

43. Pires de Miranda M, Alenquer M, Marques S, Rodrigues L, Lopes F, Bustelo XR, Simas JP. 2008. The Gammaherpesvirus $\mathrm{m} 2$ protein manipulates the Fyn/Vav pathway through a multidocking mechanism of assembly. PLoS One 3:e1654.

44. Rodrigues L, Pires de Miranda M, Caloca MJ, Bustelo XR, Simas JP. 2006. Activation of Vav by the gammaherpesvirus M2 protein contributes to the establishment of viral latency in B lymphocytes. J Virol 80:6123-35.

45. Mandal P, Krueger BE, Oldenburg D, Andry KA, Beard RS, White DW, Barton ES. 2011. A gammaherpesvirus cooperates with interferon-alpha/beta-induced IRF2 to halt viral replication, control reactivation, and minimize host lethality. PLoS Pathog 7:e1002371.

46. Shen HM, Bozek G, Pinkert CA, McBride K, Wang L, Kenter A, Storb U. 2008. and kidney. Mol Immunol 45:1883-92.

47. Gierasch WW, Zimmerman DL, Ward SL, Vanheyningen TK, Romine JD, Leib DA. 2006. Construction and characterization of bacterial artificial chromosomes containing HSV-1 strains 17 and KOS. J Virol Methods 135:197-206. 
490 48. Paden CR, Forrest JC, Moorman NJ, Speck SH. 2010. Murine gammaherpesvirus 68 LANA is essential for virus reactivation from splenocytes but not long-term carriage of viral genome. J Virol 84:7214-24.

493 49. Weck KE, Kim SS, Virgin HI, Speck SH. 1999. Macrophages are the major reservoir of latent murine gammaherpesvirus 68 in peritoneal cells. J Virol 73:3273-83.

50. Forrest JC, Paden CR, Allen RD, 3rd, Collins J, Speck SH. 2007. ORF73-null murine 
Table 1. Frequency of MHV68 latently infected cells.

500

501

\section{Table 2. Frequency of reactivation competent cells.}

\begin{tabular}{|c|c|c|c|c|c|}
\hline $\begin{array}{c}\text { Mouse } \\
\text { Strain }\end{array}$ & Virus & $\begin{array}{c}\text { Route of } \\
\text { infection }\end{array}$ & $\begin{array}{c}\text { Cell } \\
\text { population }\end{array}$ & $\begin{array}{c}\text { Day post- } \\
\text { infection }\end{array}$ & $\begin{array}{c}\text { Reactivation } \\
\text { frequency }\end{array}$ \\
\hline C57BL/6 & WT MHV68 & IN & Spleen & 16 & $1 / 2000$ \\
\cline { 2 - 6 } & M2.Stop & IN & Spleen & 16 & BLD \\
\cline { 2 - 6 } & M2.loxP & IN & Spleen & 16 & $1 / 1400$ \\
\hline CD19-Cre & WT MHV68 & IN & Spleen & 16 & $1 / 1900$ \\
\cline { 2 - 6 } & M2.loxP & IN & Spleen & 16 & $1 / 94000$ \\
\cline { 2 - 6 } & WT MHV68 & IP & Spleen & 16 & $1 / 1000$ \\
\cline { 2 - 6 } & M2.loxP & IP & Spleen & 16 & BLD \\
\hline AID-Cre & WT MHV68 & IN & Spleen & 16 & $1 / 15000$ \\
\cline { 2 - 6 } & M2.loxP & IN & Spleen & 16 & BLD \\
\cline { 2 - 6 } & WT MHV68 & IP & Spleen & 16 & $1 / 4400$ \\
\cline { 2 - 6 } & M2.loxP & IP & Spleen & 16 & $1 / 62000$ \\
\cline { 2 - 6 } & WT MHV68 & IP & PEC & 16 & $1 / 3100$ \\
\cline { 2 - 6 } & M2.loxP & IP & PEC & 16 & $1 / 1800$ \\
\hline
\end{tabular}

\begin{tabular}{|c|c|c|c|c|c|}
\hline $\begin{array}{c}\text { Mouse } \\
\text { Strain }\end{array}$ & Virus & $\begin{array}{c}\text { Route of } \\
\text { infection }\end{array}$ & $\begin{array}{c}\text { Cell } \\
\text { population }\end{array}$ & $\begin{array}{c}\text { Day post- } \\
\text { infection }\end{array}$ & $\begin{array}{c}\text { Latency } \\
\text { frequency }\end{array}$ \\
\hline C57BL/6 & WT MHV68 & IN & Spleen & 16 & $1 / 500$ \\
\cline { 2 - 6 } & M2.Stop & IN & Spleen & 16 & $1 / 1500$ \\
\cline { 2 - 6 } & M2.loxP & IN & Spleen & 16 & $1 / 350$ \\
\hline CD19-Cre & WT MHV68 & IN & Spleen & 16 & $1 / 80$ \\
\cline { 2 - 6 } & M2.loxP & IN & Spleen & 16 & $1 / 900$ \\
\cline { 2 - 6 } & WT MHV68 & IP & Spleen & 16 & $1 / 200$ \\
\cline { 2 - 6 } & M2.loxP & IP & Spleen & 16 & $1 / 300$ \\
\hline AID-Cre & WT MHV68 & IN & Spleen & 16 & $1 / 150$ \\
\cline { 2 - 6 } & M2.loxP & IN & Spleen & 16 & $1 / 1200$ \\
\cline { 2 - 6 } & WT MHV68 & IP & Spleen & 16 & $1 / 250$ \\
\cline { 2 - 6 } & M2.loxP & IP & Spleen & 16 & $1 / 1000$ \\
\cline { 2 - 6 } & WT MHV68 & IP & PEC & 16 & $1 / 500$ \\
\cline { 2 - 6 } & M2.loxP & IP & PEC & 16 & $1 / 600$ \\
\hline
\end{tabular}



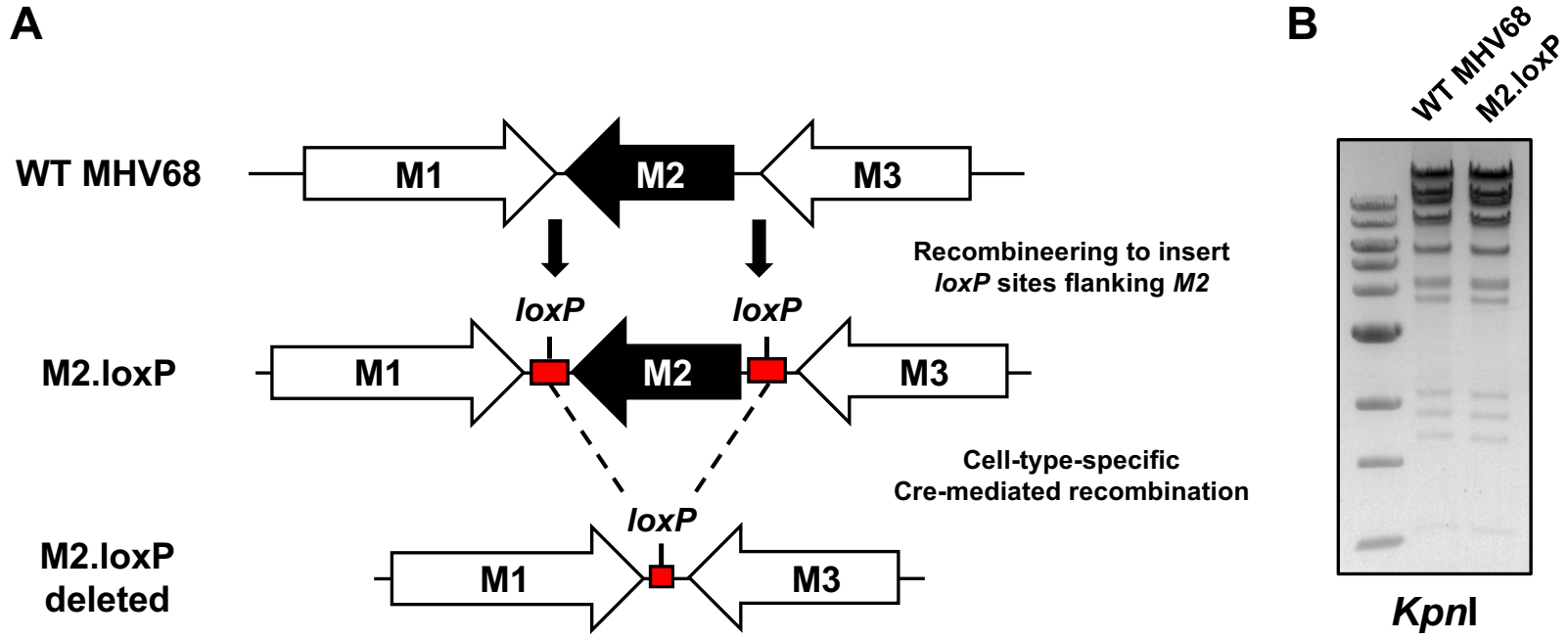

C
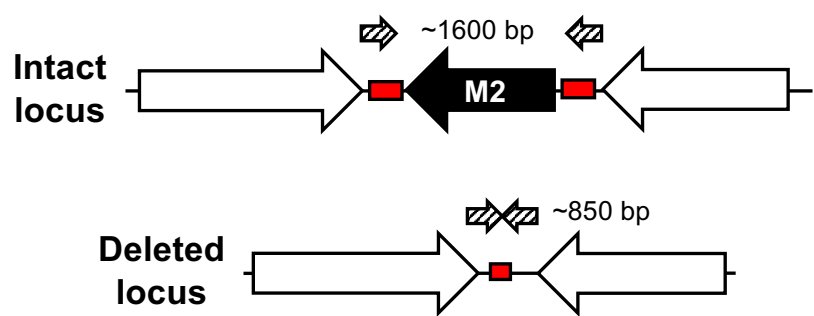

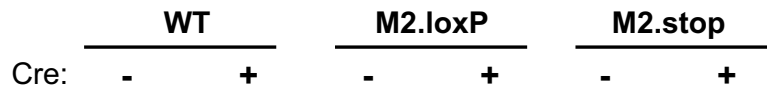

M2

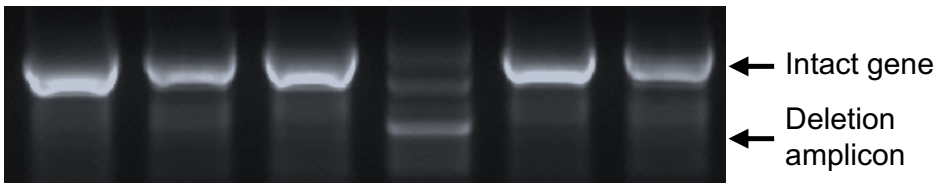

M3

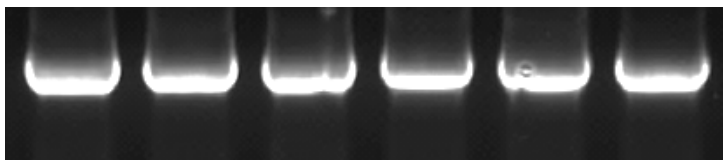

Figure 1. Development and validation of M2.IoxP MHV68. (A) Schematic depiction of the insertion of loxP sites flanking M2 in the MHV68 genome and its deletion in the presence of Cre recombinase. (B) BAC DNA was digested with the indicated restriction endonuclease, and digestion products were resolved by agarose-gel electrophoresis to evaluate the gross genetic integrity of the newly derived BAC. (C) Vero or Vero-Cre cells were infected with the indicated viruses at an MOI of 0.05 PFU/cell. Total DNA was isolated on day 4 post-infection, and PCR was performed as illustrated in the schematic to detect the intact or deleted M2 locus or the adjacent M3 locus as a control. 

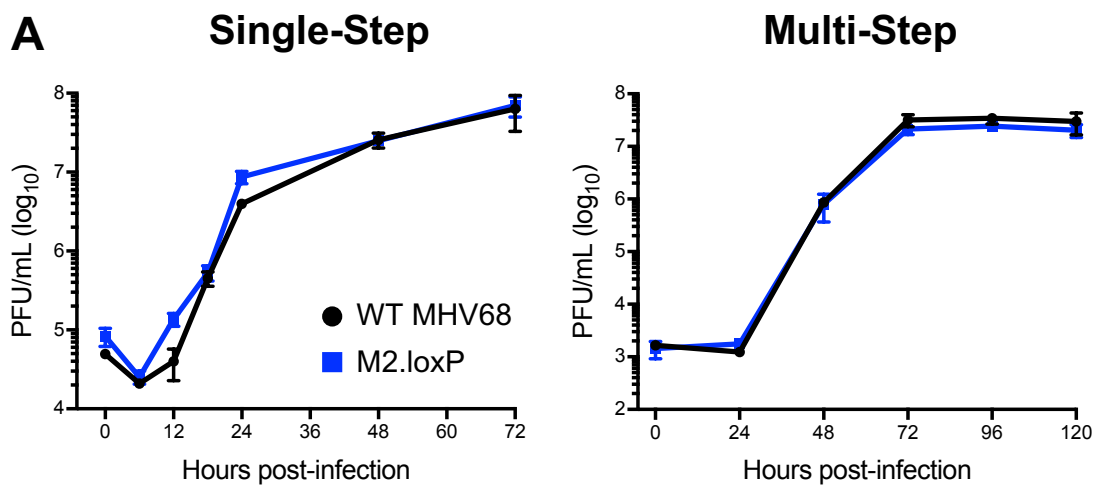

B

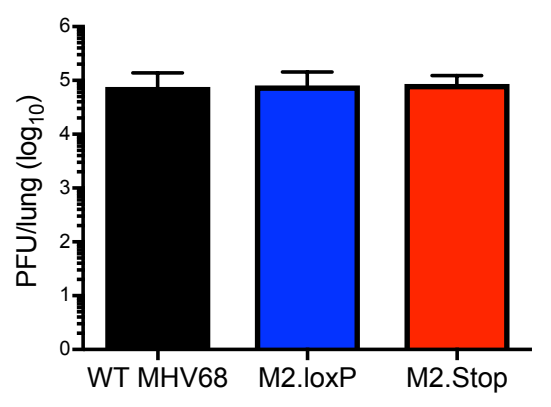

C

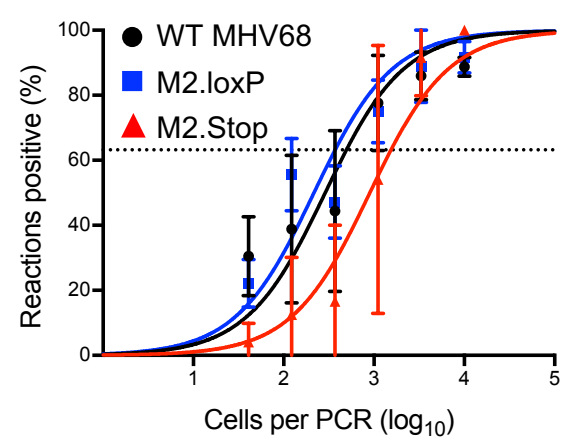

D Reactivation

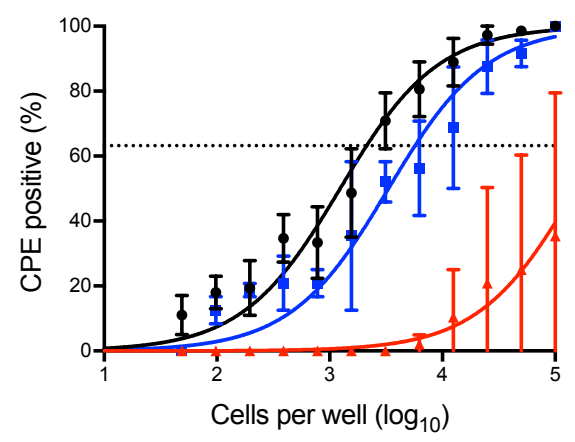

Figure 2. M2.loxP exhibits normal lytic replication and latency in C57BL/6 mice. (A) 3T12 fibroblasts were infected with WT MHV68 or M2.loxP at an MOI of 5 PFU/cell (single-step, left panel) or 0.05 PFU/cell (multi-step, right panel). Viral titers were determined by plaque assay at the indicated times post-infection. Results are means of triplicate samples. Error bars represent standard deviations. (B-D) C57BL/6 mice were infected IN with 1000 PFU of the indicated viruses. (B) Mice were sacrificed on day 7 post-infection, and viral titers in lung homogenates were determined by plaque assay. (C-D) Mice were sacrificed on day 16 post-infection. (C) Single-cell suspensions of spleen cells were serially diluted and the frequencies of cells harboring MHV68 genomes were determined using a limitingdilution PCR analysis. (D) Reactivation frequencies were determined by ex vivo plating of serially diluted cells on an indicator monolayer. Cytopathic effect was scored 2-3 weeks post-plating. Groups of 3-5 mice were pooled for each infection and analysis. Results are means of 2-3 independent infections. Error bars represent standard error of the means. 
A

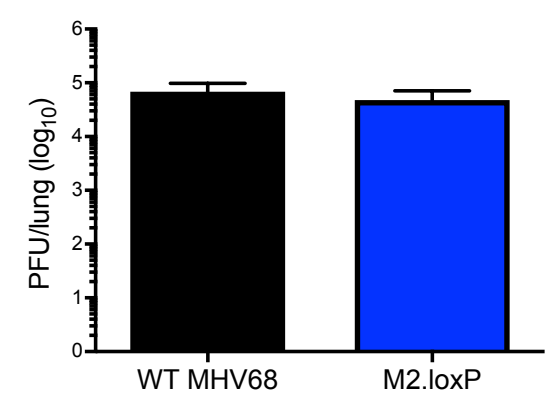

C

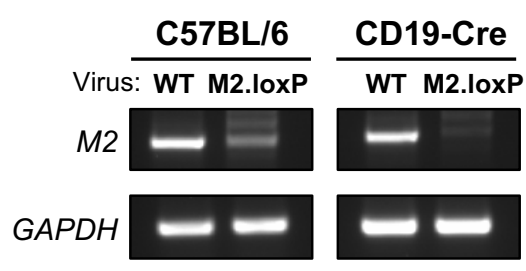

B

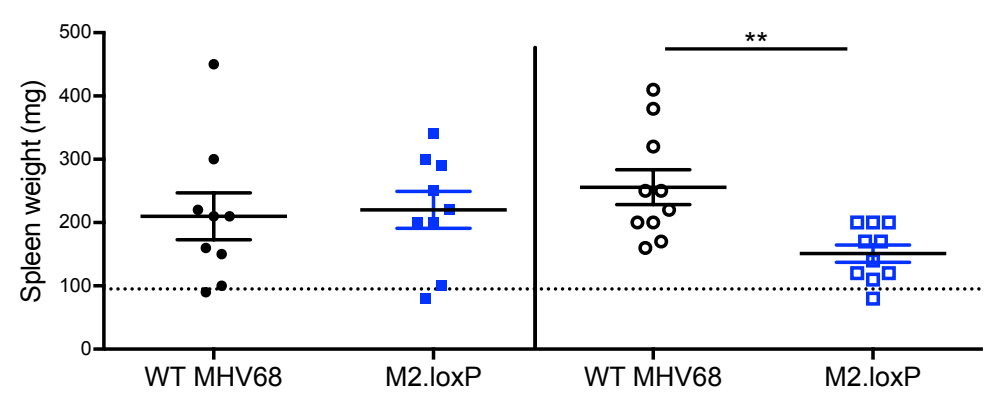

D

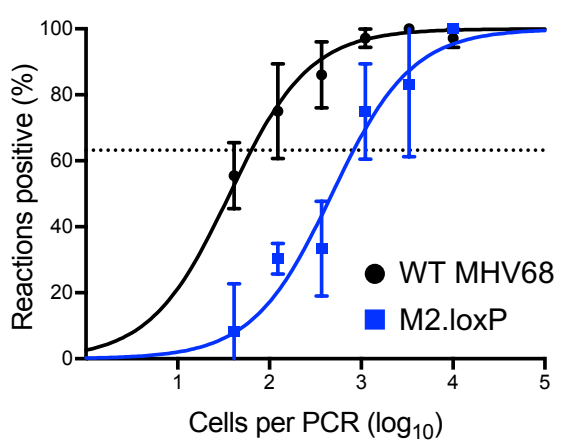

E

Reactivation

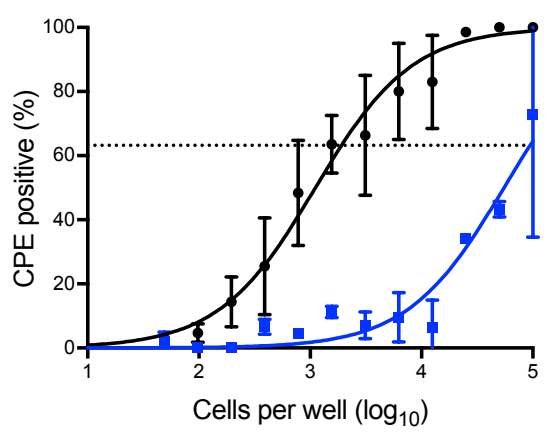

Figure 3. M2 deletion in CD19+ cells impairs MHV68 latency establishment and reactivation. CD19-Cre mice were infected IN with 1000 PFU of the indicated viruses. (A) Mice were sacrificed on day 7 post-infection, and viral titers in lung homogenates were determined by plaque assay. (B-E) Mice were sacrificed on day 16 post-infection. (B) Spleens were harvested and weighed as a measure of splenomegaly. The dashed line indicates the average mass of spleens from mock-infected mice. Each dot represents one mouse. Spleen weights from C57BL/6 mice infected in Figure 2 are shown for comparison. (C) DNA was isolated from infected spleens, and PCR was performed to evaluate the integrity of the M2 locus. Cellular GAPDH serves as an amplification control. (D) Single-cell suspensions of spleen cells were serially diluted, and the frequencies of cells harboring MHV68 genomes were determined using a limitingdilution PCR analysis. (E) Reactivation frequencies were determined by ex vivo plating of serially diluted cells on an indicator monolayer. Cytopathic effect was scored 2-3 weeks post-plating. Groups of 3-5 mice were pooled for each infection and analysis. Results are means of 2-3 independent infections. Error bars represent standard error of the means. ${ }^{* *}$ denotes $p<0.01$ in a two-tailed student's t-test. 
A

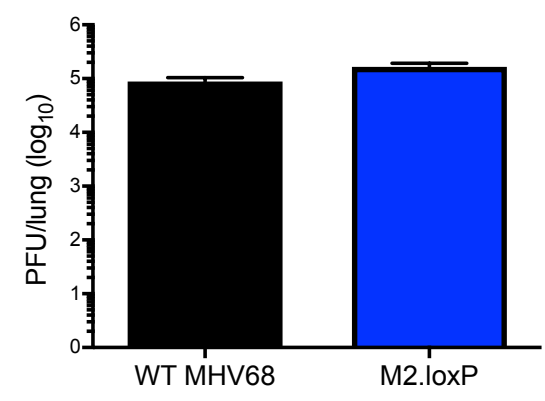

B

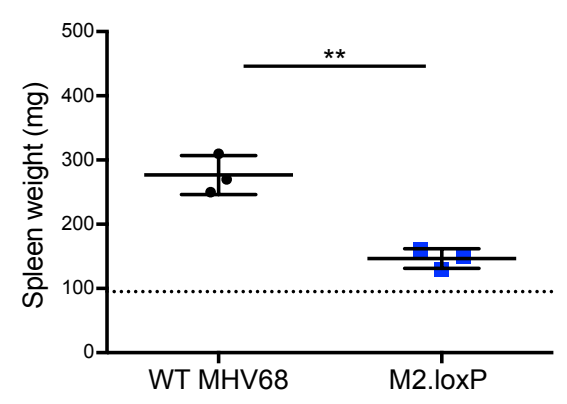

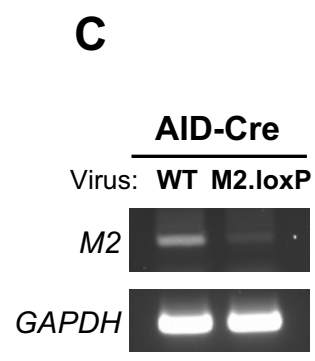

D

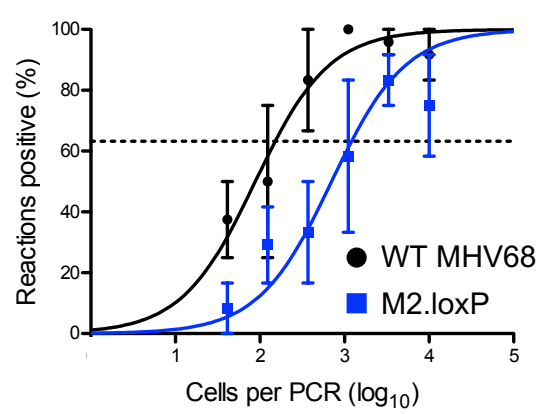

E Reactivation

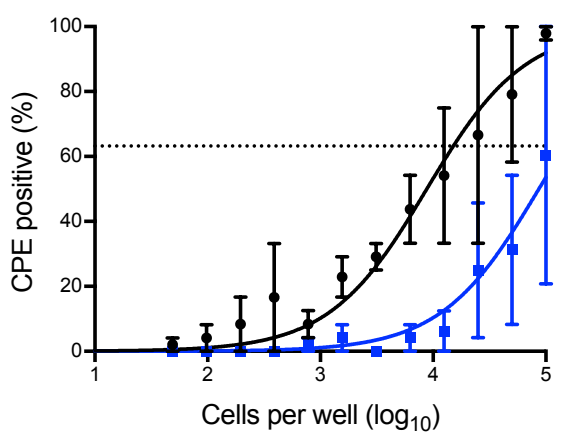

Figure 4. M2 deletion in AID-expressing cells impairs MHV68 latency and reactivation. AID-Cre mice were infected IN with 1000 PFU of the indicated viruses. (A) Mice were sacrificed on day 7 post-infection, and viral titers in lung homogenates were determined by plaque assay. (B-E) Mice were sacrificed on day 16 post-infection. (B) Spleens were harvested and weighed as a measure of splenomegaly. The dashed line indicates the average mass of spleens from mock-infected mice. Each dot represents one mouse. (C) DNA was isolated from infected spleens, and PCR was performed to evaluate the integrity of the M2 locus. Cellular GAPDH serves as an amplification control. (D) Single-cell suspensions of spleen cells were serially diluted, and the frequencies of cells harboring MHV68 genomes were determined using a limiting-dilution PCR analysis. (E) Reactivation frequencies were determined by ex vivo plating of serially diluted cells on an indicator monolayer. Cytopathic effect was scored 2-3 weeks post-plating. Groups of 3-5 mice were pooled for each infection and analysis. Results are means of 2-3 independent infections. Error bars represent standard error of the means. ${ }^{* *}$ denotes $p<0.01$ in a two-tailed student's t-test. 

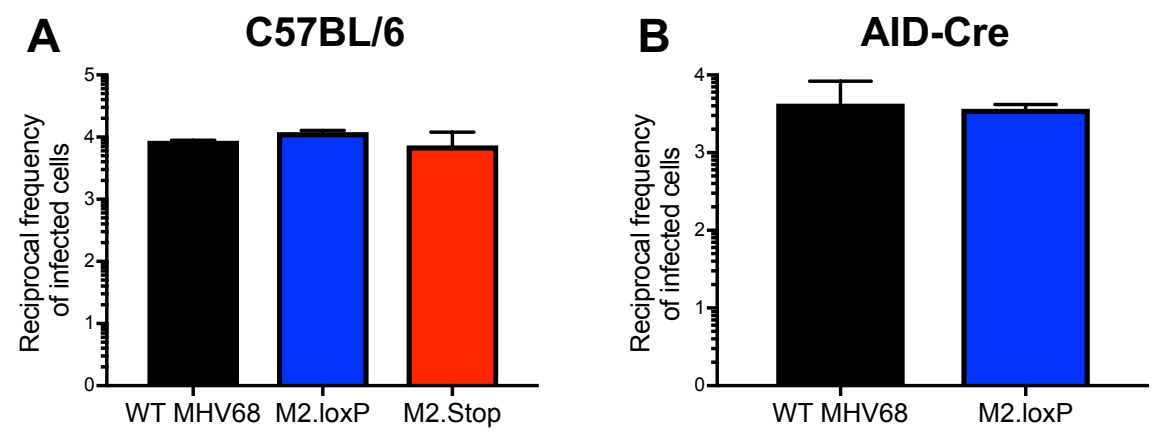

Figure 5. M2 expression is not required for MHV68 colonization of draining lymph nodes. (A) C57BL/6 or (B) AID-Cre mice were infected IN with 1000 PFU of indicated virus. Single-cell suspensions of MLNs were serially diluted and the frequencies of cells harboring MHV68 genomes were determined using a limiting-dilution PCR analysis. Reciprocal frequencies shown. Groups of 3-5 mice were pooled for each infection and analysis. Results are means of $2-3$ independent infections. Error bars represent standard error of the means. 

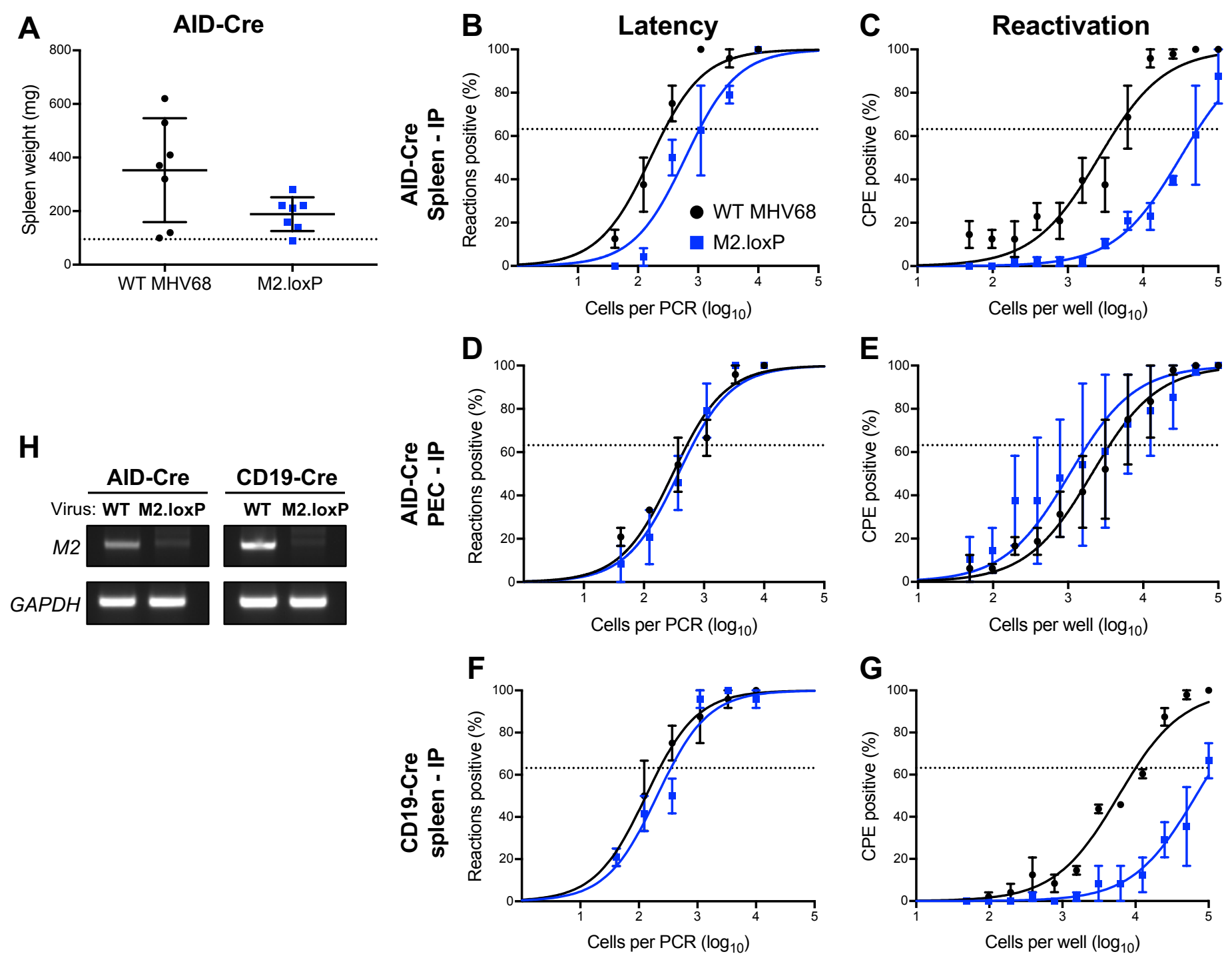

Figure 6. M2 expression in $\mathrm{B}$ cells is necessary for reactivation, but not latency establishment, following direct lymphoid tissue infection. AID-Cre (A-E, H) or CD19-Cre (F-H) mice were infected IP with 1000 PFU of the indicated virus and sacrificed on day 16 post-infection. (A) Spleens were harvested and weighed as a measure of splenomegaly. The dashed line indicates the average mass of spleens from mock-infected mice. Each dot represents one mouse. Single-cell suspensions of spleen cells $(B, F)$ or PECs (D) were serially diluted, and the frequencies of cells harboring MHV68 genomes were determined using a limiting-dilution PCR analysis. Reactivation frequencies of splenocytes (C, $\mathrm{G}$ ) or PECs (E) were determined by ex vivo plating of serially diluted cells on an indicator monolayer. Cytopathic effect was scored 2-3 weeks post-plating. (H) DNA was isolated from infected spleens of the indicated mouse strain, and PCR was performed to evaluate the integrity of the M2 locus. Cellular GAPDH serves as an amplification control. Groups of 3-5 mice were pooled for each infection and analysis. Results are means of 2-3 independent infections. Error bars represent standard error of the means. ns denotes non-significant in a two-tailed student's t-test. ${ }^{* *}$ denotes $p<0.01$ in a two-tailed student's t-test. 


\section{A}

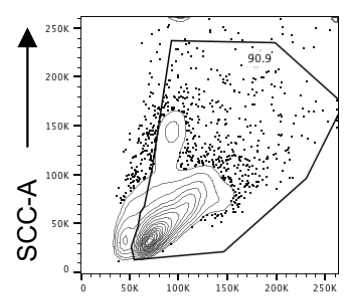

FSC-A

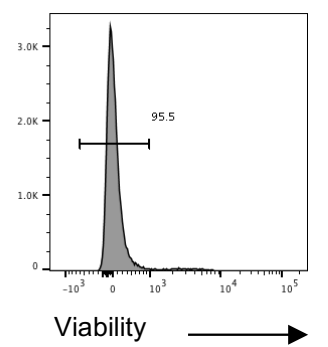

Unsorted

B

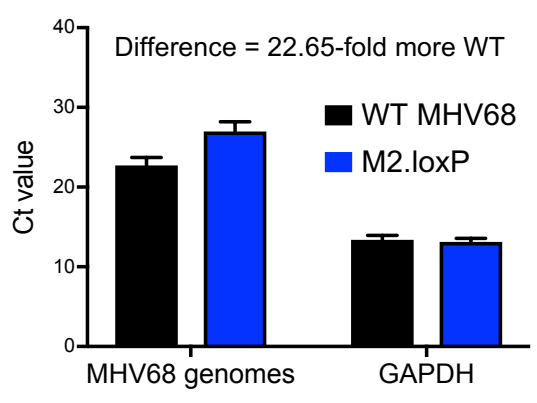

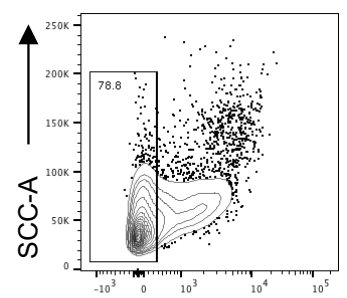

Dump

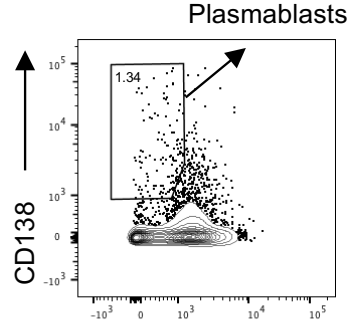

CD38

\section{Plasmablast}

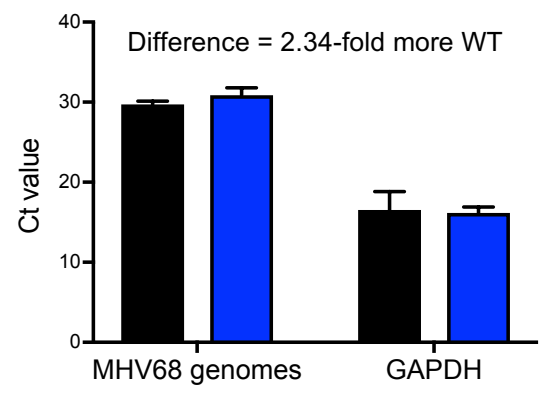

Figure 7. Loss of M2 in AID-expressing cells does not reduce infection of plasmablasts. AID-Cre mice were infected IP with 1000 PFU of the indicated virus and were sacrificed on day 14 post-infection. Spleens were harvested and stained as indicated for FACS-based sorting. Representative plots in panel (A) demonstrate the gating strategy used for sorting of $\mathrm{CD} 138^{+} \mathrm{CD} 38^{\mathrm{lo}}$ plasmablasts. (B) DNA was isolated from unsorted splenocytes or sorted plasmablasts and qPCR was performed on $50 \mathrm{ng}$ of DNA to detect MHV68 genomes or cellular GAPDH as a control. Fold difference in MHV68 genome quantities was determined using the $\Delta \Delta \mathrm{Ct}$ method. 

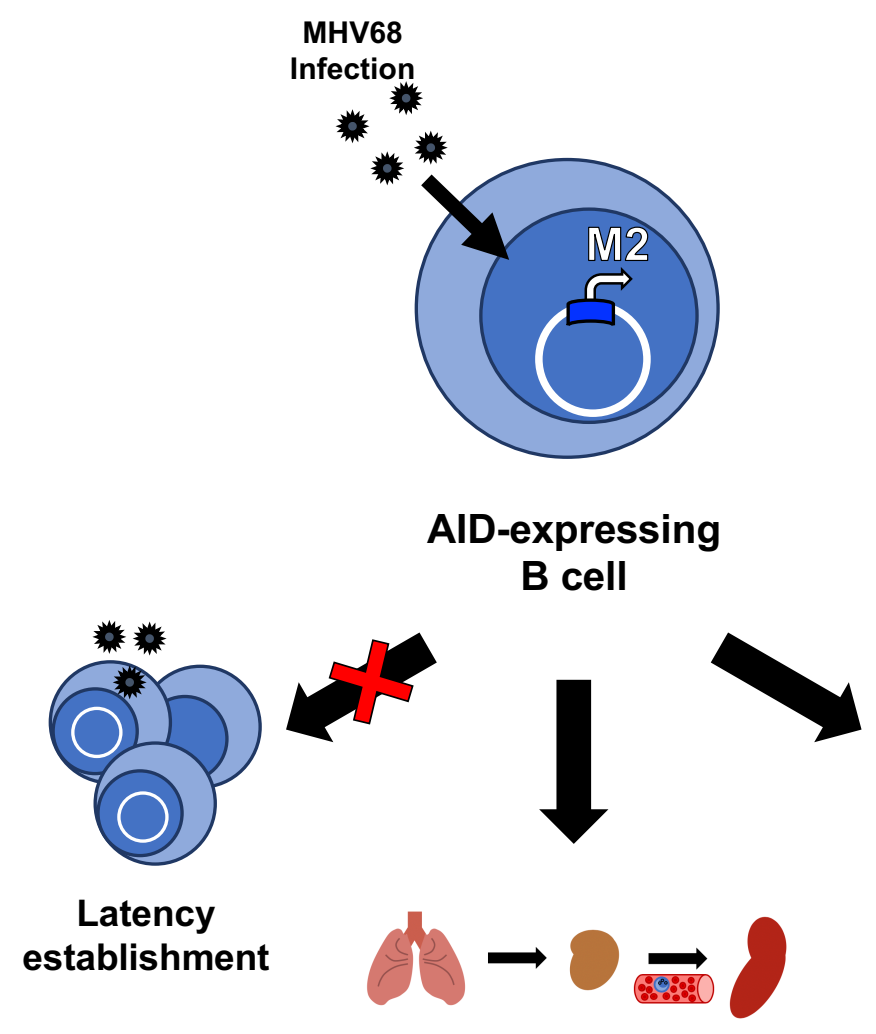

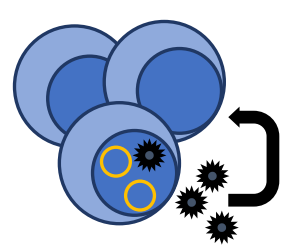

Viral reactivation

Host colonization

Figure 8. Model of cell-type-specific requirements for M2 in MHV68 latency. The majority of M2-null MHV68 phenotypes are recapitulated by specific $M 2$ deletion in AID-expressing B cells. These phenotypes overlap with those observed when $\mathrm{M} 2$ is deleted in all $\mathrm{CD} 19^{+} \mathrm{B}$ cells. $\mathrm{M} 2$ is not directly required for viral infection of and apparent initial expansion in lymphoid tissues, but plays a role in facilitating dissemination within the host and reactivation during early latency time points. Results of plasma cell infection were inconclusive. 